\title{
Lexis
}

Journal in English Lexicology

HS 2 | 2010

Theoretical Approaches to Linguistic (Im)politeness

\section{La fausse hiérarchisation entre information nouvelle et information ancienne à l'épreuve des modèles théoriques de la politesse}

Rudy Loock

\section{(2) OpenEdition}

Journals

Édition électronique

URL : http://journals.openedition.org/lexis/830

DOI : $10.4000 /$ lexis. 830

ISSN : 1951-6215

Éditeur

Université Jean Moulin - Lyon 3

Référence électronique

Rudy Loock, « La fausse hiérarchisation entre information nouvelle et information ancienne à l'épreuve des modèles théoriques de la politesse », Lexis [En ligne], HS 2 | 2010, mis en ligne le 06 septembre 2010, consulté le 01 mai 2019. URL : http://journals.openedition.org/lexis/830 ; DOI : 10.4000/ lexis.830

\section{(c) (1) () $\Theta$}

Lexis is licensed under a Creative Commons Attribution-NonCommercial-NoDerivatives 4.0 International License. 


\title{
La fausse hiérarchisation entre information nouvelle et information ancienne à l'épreuve des modèles théoriques de la politesse
}

Rudy Loock ${ }^{1}$

\section{Résumé}

Cet article propose d'étudier la façon dont l'apport d'information nouvelle à un destinataire peut représenter un acte d'impolitesse et comment cet acte menaçant pour la face de l'autre (FTA selon le modèle de Brown \& Levinson [1978], [1987]) peut être adouci par l'emploi de structures hiérarchisantes comme notamment la proposition subordonnée relative appositive ou par l'emploi d'adoucisseurs tels que you know ou as you know. Nous soulevons également les questions de la communication de l'acte de politesse lui-même, ainsi que de la conception de la politesse comme un continuum. A travers des exemples oraux attestés relevant de cette stratégie, l'objectif final de cet article est de confronter les données étudiées aux différents modèles théoriques et outils conceptuels proposés par la littérature.

Mots-clés : relatives appositives - apposition - politesse - statut informationnel - pertinence - adoucisseur

$$
* * *
$$

\begin{abstract}
The aim of this article is to study how the ways in which new information is conveyed to a hearer can represent an act of impoliteness and how this face-theatening act or FTA (Brown \& Levinson [1978, 1987]) can be softened by the use of hierarchizing structures, in particular appositive relative clauses, or by the use of softeners such as you know or as you know. We also tackle questions relating to whether the act of politeness itself must be conveyed and whether (im)politeness can be seen as a continuum. Using attested spoken examples illustrating such strategies, the ultimate aim of this article is to confront the data with the theoretical models and conceptual tools that the literature on the subject has to offer.
\end{abstract}

Keywords: appositive relative clauses - apposition - politeness - informational status relevance - softener

\footnotetext{
${ }^{1}$ Université C. de Gaulle Lille 3 \& UMR 8163 Savoirs, Textes, Langage du CNRS : rudy.loock@univ-lille3.fr ; http://stl.recherche.univ-lille3.fr/sitespersonnels/loock
} 


\section{Introduction}

Le but de cet article est de montrer comment la simulation d'une hiérarchie informationnelle entre un contenu présenté comme étant nouveau pour le(s) destinataire(s) et un contenu présenté comme étant possiblement connu relève d'une stratégie de politesse au sens théorique du terme (politesse 2 selon la distinction de Watts [2003]). Cette différence simulée de statut informationnel s'exprime alors au moyen d'une structure hiérarchisante, parmi lesquelles la proposition subordonnée relative appositive (désormais PRA), telle qu'illustrée en (1) par opposition à la PRD ou proposition subordonnée relative déterminative (2) au sein d'une dichotomie devenue classique, et sur laquelle nous souhaitons concentrer notre analyse ici :

(1) The students, who study linguistics, are polite.

(2) The students who study linguistics are polite.

L'article est organisé de la façon suivante. Dans un premier temps, nous expliquons comment, dans le cadre d'un travail sur les PRA, nous avons été amenés à nous pencher sur la question de la politesse dès lors que certains exemples semblaient contrevenir aux stratégies discursives que nous avions définies pour cette structure. Dans un deuxième temps, nous plaçons notre analyse de ces données « déviantes » dans le cadre théorique proposé par Brown \& Levinson [1978, 1987] afin d'expliquer comment l'emploi de la PRA peut permettre d'éviter de commettre un FTA (Face-Threatening Act) tel que défini par ces chercheurs. Nous montrons néanmoins les limites de ce modèle, notamment s'agissant de la communication de l'acte de politesse lui-même, et faisons appel aux travaux d'autres chercheurs sur la question. $\mathrm{Au}$ travers d'exemples attestés tirés notamment de la section orale du corpus électronique $\mathrm{ICE}_{\mathrm{GB}}{ }^{2}$, nous étudions également la présence d'adoucisseurs (softeners) tels que définis par Brown \& Levinson, en particulier la séquence (as) you know. Enfin, dans une troisième section, nous soulevons la question de l'existence d'un continuum en fonction du statut informationnel réel, cette fois, de l'information véhiculée, ainsi que du choix de la structure hiérarchisante parmi les différentes structures concurrentes possibles.

\section{Point de départ}

\subsection{Les fonctions discursives des propositions relatives appositives}

C'est dans le cadre d'un travail sur les propositions subordonnées relatives appositives en anglais (PRA) que s'est posée la question que nous soulevons ici. Partant du constat que la fonction en discours de la PRA était traditionnellement définie en creux par rapport à la PRD, également appelée relative restrictive dans la mesure où elle vient « restreindre » le référent de l'antécédent, nous avons proposé, corpus de données attestées écrites et orales à l'appui, une taxonomie de fonctions discursives pour cette sous-catégorie de subordonnées relatives peu étudiée en dehors du débat syntaxique sur l'opposition PRA-PRD (Loock [2003], [2005], [2007], [2010]). Nous avons ainsi dégagé trois fonctions discursives majeures de la PRA, reprises dans le schéma ci-dessous et illustrées par les exemples (3), (4) et (5).

\footnotetext{
${ }^{2}$ Le corpus ICE-GB (International Corpus of English, British Component) contient 1 million de mots tirés de documents écrits et oraux datant des années 1990 (http://www.ucl.ac.uk/english-usage/projects/ice-gb/).
} 


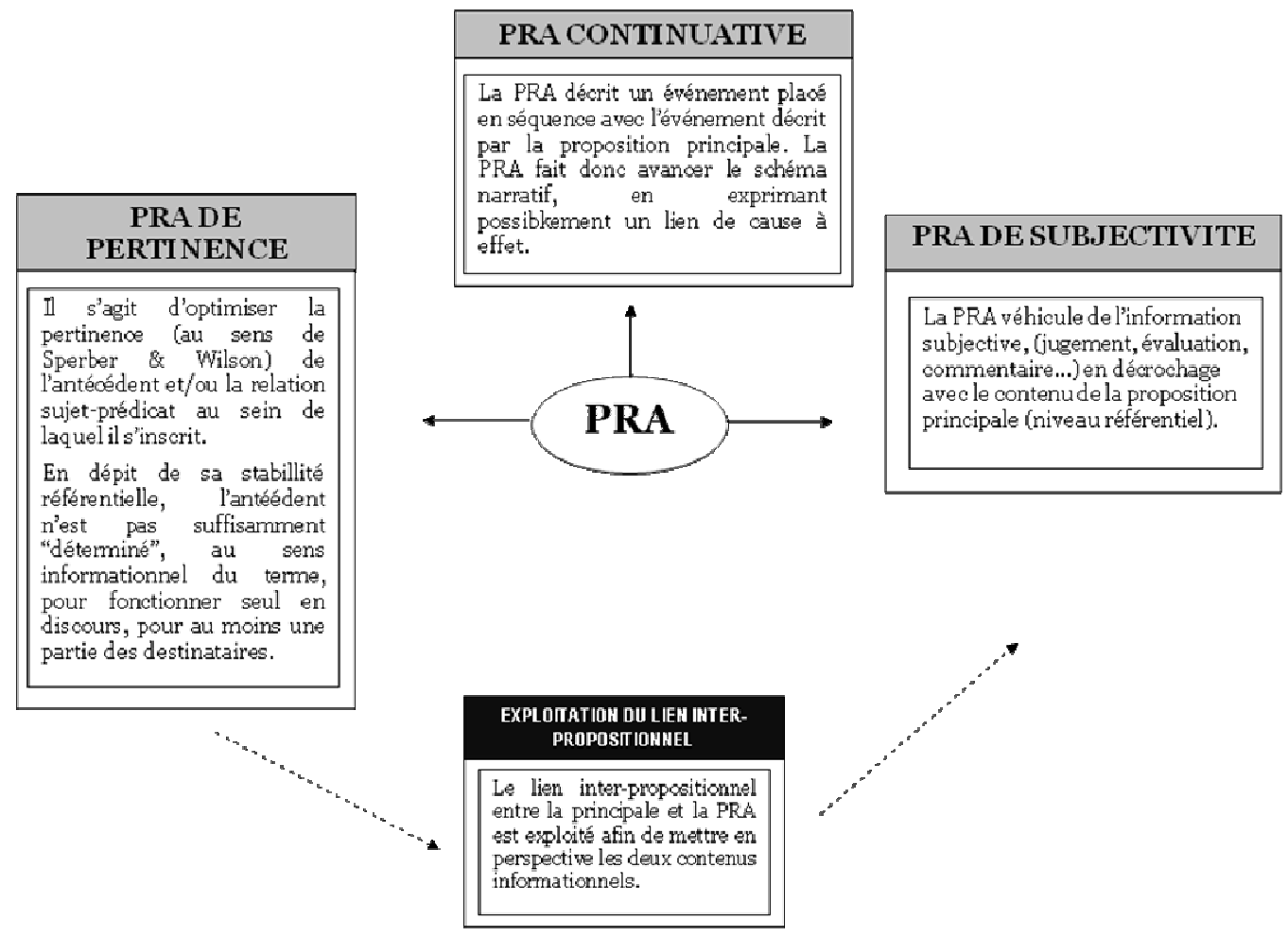

Figure 1. Les fonctions discursives des subordonnées relatives appositives

(3) So we asked a man, who shrugged his shoulders and disappeared into a nearby shop. [PRA continuative]

(4) It is hard to square his action with the Energy Policy and Conservation Act, which authorizes the president to tap the reserve. [PRA de pertinence]

(5) This incredible spirit = which Chelsea so clearly lack - is summed up beautifully by Gemmill, who has been unable to command a regular plane and has also been a target for some of the fans on his rare appearances. [PRA de subjectivité]

\subsection{Le nivellement de l'espace cognitive partagé}

Parmi les stratégies conversationnelles relevant de l'emploi de la PRA de pertinence, sur laquelle nous souhaitons nous attarder ici, nous avons dégagé l'opération de nivellement de l'espace cognitif partagé. L'énonciateur, lorsqu'il est notamment face à des destinataires multiples aux états de connaissance par définition variables, véhicule au moyen d'une PRA de l'information qu'il juge pertinente et non connue par au moins une partie des destinataires. Le cas le plus prototypique de ce type de stratégie est le cas du journaliste s'adressant à un lectorat par essence hétérogène. Ainsi, si l'on se réfère à la taxonomie de Prince [1981, 1992] que nous avons étendue concernant le caractère nouveau ou ancien de l'information véhiculée $^{3}$, dans les exemples (6) et (7) ci-dessous, l'information véhiculée par la PRA est

\footnotetext{
${ }^{3}$ Prince opère une distinction cruciale entre le caractère nouveau/ancien de l'information du point de vue du discours (discourse new/old) et du point de vue des connaissances supposées du/des destinataire(s) (hearer new/old). Ce à quoi elle ajoute le statut des inferrables pour qualifier les informations dont l'existence peut être inférée par les destinataires. De notre côté, nous avons ajouté une sous-catégorie supplémentaire, les
} 
nouvelle du point de vue du discours (discourse new) mais indéterminable du point de vue des connaissances supposées (hearer new/old?), certains destinataires connaissant la nature de la conférence de Dayton de 1995 ou le rôle des globules rouges et des plaquettes, tandis que d'autres non. L'énonciateur est bien entendu incapable, dans le cadre d'une communication in absentia, de déterminer avec certitude les connaissances des destinataires :

(6) At the Dayton conference in 1995, which ended the war in Bosnia, Milosevic was still central to American diplomatic efforts.

(7) Blood from donors is usually split into its components, such as red cells, which carry oxygen; plasma, with white cells that help fight infection; and platelets, which aid clotting.

Il existe alors une hiérarchisation entre la proposition principale, qui est nouvelle du point de vue du discours et des connaissances partagées (discourse new/hearer new) et la PRA, qui est nouvelle du point de vue du discours mais de statut informationnel indéterminable du point de vue des connaissances supposées du/des destinataire(s) par l'énonciateur. Il y a alors une disjonction entre ce dont on parle (information nouvelle, foreground) et de l'information dite "périphérique » (background, not at-issue) dont le but est d'optimiser la pertinence de l'ensemble, ici en nivelant l'espace cognitif partagé entre l'énonciateur et ses destinataires hétérogènes. Ceci permet alors d'établir un compromis entre le destinataire initié et le destinataire non-initié.

La PRA n'est pas la seule structure hiérarchisante permettant de véhiculer de l'information de statut informationnel indécidable à destination de destinataires hétérogènes ou d'un destinataire dont les connaissances ne sont pas suffisamment connues par l'énonciateur. D'autres structures remplissent le même type de fonctions et entrent en compétition avec la PRA (Loock [2005], [2010]). Parmi ces allostructures, ou structures concurrentes, figurent le syntagme nominal apposé (8), la proposition adverbiale (9), le prémodifieur non-restrictif (10), la parenthétique phrastique (11) ou encore le syntagme nominal co-référentiel dans le cadre de la reprise anaphorique (12) :

(8) Mullah Mohammed Omar, the reclusive spiritual leader of the Taliban, broadcast to the nation that Afghanistan had nothing to fear from the "cowardly" United States.

(9) [A]ll British-donated blood has been stripped of its white cells, because they are thought most likely to carry the infectious prion particles.

(10) Former president of the United States Bill Clinton will attend an international seminar on AIDS and SARS.

(11) Hassan Rohani - he is the chief Iranian negotiator - said after three hours of talks in Geneva yesterday that the Europeans had until the end of July to come up with a more concrete offer.

(12) $A T \& T_{i}$ will announce today plans to sell Internet phone service to consumers and to more businesses. David Dorman, the CEO of the No. 1 long-distance carrier $_{i}$, speaking at an investor conference, will detail the opening phase.

Dans tous ces cas de figure, la promotion de l'information au foreground est pragmatiquement inacceptable - trois exemples suffiront :

indéterminables pour qualifier l'information dont le statut informationnel pour le(s) destinataire(s) ne peut être déterminé par l'énonciateur (voir Loock [2010: 84-86]). 
(6a) At the Dayton conference in 1995, Milosevic was still central to American diplomatic efforts. It ended the war in Bosnia.

(8a) \#Mullah Mohammed Omar is the reclusive spiritual leader of the Taliban.

He broadcast to the nation that Afghanistan had nothing to fear from the "cowardly" United States.

(10a) \#Bill Clinton is the former president of the United States. He will attend an international seminar on AIDS and SARS.

\subsection{Contre-exemples apparents}

Or, un certain type d'exemples semble contrevenir à cette hiérarchisation des contenus. Considérons l'exemple suivant, fabriqué pour la circonstance et néanmoins accompagné d'un contexte avant de nous consacrer exclusivement à des exemples oraux attestés.

(13) [Contexte : A informe B, son supérieur hiérarchique, de la venue de Shirin Ebadi. A sait que B ne sait pas qui elle est.]

Shirin Ebadi, who won the 2003 Nobel Peace Prize, has just arrived in town.

Dans cet exemple, l'information de la proposition principale, mais aussi l'information de la PRA, sont nouvelles pour le destinataire à la fois du point de vue du discours et du point de vue des connaissances partagées (discourse new/hearer new selon la taxonomie de Prince), puisque l'énonciateur sait que son destinataire ne connaît pas l'information. L'emploi d'une structure hiérarchisante, qui fait passer au second plan, en périphérie, l'information sur l'identité de Shirin Ebadi donne un statut autre à cette information. En particulier, en basculant vers l'arrière-plan informationnel, elle acquiert, contrairement à l'information de la prédication principale, un statut informationnel qui devient indécidable, l'indécidabilité quant au statut informationnel véhiculé étant l'un des déclencheurs linguistiques pour l'emploi d'une PRA de pertinence ${ }^{4}$. Il y a donc au final une fausse hiérarchisation puisque les deux contenus informationnels sont en réalité discourse new/hearer new ${ }^{5}$, la PRA permettant de présenter l'information comme étant potentiellement connue (cf. le «simple rappel», mentionné dans bon nombre de grammaires pédagogiques), plutôt que comme de l'information nouvelle.

En comparaison, les énoncés (13a) et (13b) paraissent par conséquent intuitivement moins polis, puisqu'ils mettent les deux informations (arrivée et identité de Shirin Ebadi) sur le même plan informationnel :

(13a) \#Shirin Ebadi has just arrived in town. She is the 2003 Nobel Peace Prize winner.

(13b) \#Shirin Ebadi is the 2003 Nobel Peace Prize winner. She has just arrived in town.

Avec (13a) et (13b) l'énonciateur semble présupposer l'ignorance de son destinataire, d'où un possible effet d'impolitesse.

\footnotetext{
${ }^{4}$ À l'inverse, l'information véhiculée par les autres types de PRA (continuatives/subjectivité) est discourse new/hearer new de façon systématique.

${ }^{5}$ Ceci ne revient cependant pas à dire que toute hiérarchisation est le résultat d'un décrochage lié aux statuts informationnels. Ainsi, dans les textes de fiction, où tout contenu informationnel est, sauf cas particuliers, discourse new/hearer new, l'auteur exerçant un contrôle total sur les connaissances de ses lecteurs, la PRA existe pourtant et résulte d'une hiérarchisation des contenus qui est indépendante du statut informationnel de l'information véhiculée.
} 
Face à ce type de données, qui semblent pouvoir s'expliquer par des phénomènes de politesse, il convient de se poser les questions suivantes :

(i) Comment décrire cette forme de politesse ?

(ii) Quels outils d'analyse utiliser pour décrire ces exemples qui semblent contrevenir à la définition de la stratégie d'optimisation de la pertinence par nivellement de l'espace cognitif partagé ?

(iii) Comment mettre au jour cette stratégie ?

(iv) Est-elle perçue par le(s) destinataire(s)?

(v) Existe-t-il différents degrés de politesse ?

C'est à ces différentes questions que cet article propose d'apporter des réponses, en partant du modèle théorique le plus connu, celui de Brown \& Levinson [1978, 1987].

\section{L'apport d'information nouvelle dans le modèle de Brown \& Levinson}

\subsection{L'apport d'information nouvelle comme FTA}

Afin d'étudier les phénomènes de politesse tels que décrits dans la partie précédente, nous avons souhaité nous placer dans le cadre théorique proposé par Brown \& Levinson [1978, 1987]. Ce modèle théorique, qui considère la politesse comme un travail de préservation des faces (face work) telles que définies dans les travaux de Goffman [1967] notamment, a permis de formaliser le concept de politesse au sens linguistique du terme. Si les critiques de ce modèle n'ont pas manqué (et nous ne ferons pas exception), la définition de la politesse qu'ils proposent s'avère particulièrement opérante.

Selon Brown \& Levinson, chaque énoncé est potentiellement menaçant pour la «face » du destinataire. Tout un chacun a donc un face want, c'est-à-dire un territoire et une face qu'il s'attache à préserver. L'apport d'information nouvelle peut représenter un acte menaçant pour la face du destinataire (face-threatening act ou FTA) dans la mesure où l'énonciateur présuppose alors l'ignorance de celui-ci. La menace pour la face de l'autre est d'autant plus importante qu'il existe une certaine distance, soit de pouvoir $(\mathrm{P})$ soit sociale $(\mathrm{S})$ entre les deux participants à l'interaction ( $\mathrm{S}$, speaker et $\mathrm{H}$, hearer), à quoi Brown \& Levinson ajoutent également la nature, dans la culture en question, de l'action commise (R, ranking, défini comme suit: "absolute ranking of impositions in the particular culture"). La somme de ces paramètres confère alors un certain poids ( $\mathrm{W}$, weightiness) au FTA $(\mathrm{Wx}=\mathrm{D}(\mathrm{S}, \mathrm{H})+\mathrm{P}(\mathrm{H}, \mathrm{S})$ $+\mathrm{Rx}$ ), poids qui conditionnera les choix linguistiques de l'énonciateur afin de minimiser le FTA.

Ainsi, l'apport d'information nouvelle est un FTA d'autant plus important qu'il existe une forte relation de pouvoir $(\mathrm{P}+)$ ou une forte de distance sociale $(\mathrm{D}+)$ entre les participants : dire à son supérieur hiérarchique ou à son meilleur ami que Shirin Ebadi est la lauréate du Prix Nobel de la Paix de 2003 alors que l'information principale est son arrivée en ville n'est pas la même chose. Si la présupposition d'ignorance peut être non menaçante entre des personnes dans une relation proche et entre qui aucune relation de pouvoir n'existe, elle peut en revanche être considérée comme très menaçante pour la face d'un supérieur hiérarchique, venant d'un subalterne.

Le type d'information lui-même, et en particulier son potentiel en tant qu'information nouvelle, modifient également le poids du FTA. Considérons l'exemple (14) :

(14) Then I became interested in something called Tai Chi $<,>$ which is a a Chinese movement form $<,>$ (ICE-GB s 1a-004 072) 
Donner au destinataire l'information [Tai Chi/be a Chinese movement form] représente potentiellement un FTA plus important que l'information [S. Ebadi/be the 2003 Nobel Peace Prize winner] dans la mesure où l'information concernant l'identité de Shirin Ebadi est potentiellement moins connue que la nature du Tai Chi, dont tout le monde ou presque a plus ou moins une vague idée. Le statut informationnel connu, ou encore la notoriété (voir section 3.1.), relèverait alors du paramètre $\mathrm{R}$ (ranking) tel que défini dans la théorie de Brown \& Levinson.

Le choix d'une structure hiérarchisante telle la PRA permet à l'énonciateur de minimiser le FTA (Brown \& Levinson parlent alors de redressive action). Ce choix structurel permet de sauver la face du destinataire en ne présupposant pas l'ignorance de ce dernier mais en présentant l'information comme étant possiblement connue (soit hearer new, soit hearer old), donc en ne présupposant rien quant à un statut informationnel exclusivement nouveau. Si l'on se réfère à la situation illustrée par l'exemple (13), l'énonciateur établit ainsi une fausse hiérarchisation en présentant de l'information dont il sait qu'elle n'est pas connue (hearer new) comme l'étant possiblement.

Comme l'emploi de Est-ce que tu pourrais fermer la fenêtre s'il te plaît au lieu de ferme la fenêtre, qui serait pourtant plus économe (Kerbrat Orecchioni [2005: 200]), ce choix structurel représente alors une déviation vis-à-vis de la façon la plus directe de fournir de l'information nouvelle comme l'emploi d'une proposition indépendante par exemple (13a-b supra) ou encore (14a) :

\section{(14a) Then I became interested in something called Tai Chi $<,>$ It's a a Chinese movement form}

C'est cette même idée de « déviation » que l'on retrouve dans la définition de la politesse telle que formalisée par Brown \& Levinson :

Politeness is then a major source of deviation from [the] rational efficiency [of speaking in accordance with Grice's Maxims] ${ }^{6}$. [Brown \& Levinson 1987 : 95]

La stratégie linguistique du choix de la structure hiérarchisante (PRA) apparaît alors comme le compromis qui permettra de protéger la face du destinataire. Si présenter l'information comme étant nouvelle (hearer new) peut représenter un FTA qu'il convient de modérer, ne pas véhiculer l'information (13c-14b) peut en effet également représenter un FTA :

(13c) Shirin Ebadi has just arrived in town.

(14b) Then I became interested in something called Tai Chi. ${ }^{7}$

\subsection{Politesse positive vs. politesse négative}

Brown \& Levinson [1987: 62] ont défini plusieurs types de politesse et opèrent notamment une distinction entre la politesse positive et la politesse négative. La politesse

\footnotetext{
${ }^{6}$ Nous soulignons.

${ }^{7}$ Pour preuve, nous pouvons citer ici les cas fréquents d'interruptions de journalistes de radio ou de télévision (B) lorsque la personne interviewée (A) utilise des noms propres de personne dont le référent et/ou la fonction peut ne pas être connue de tous les destinataires (auditeurs/téléspectateurs), comme dans l'exemple attesté suivant :

(i) A : Tout ceci est arrivé avant que Patrick de Carolis n'intervienne...

B : ...qui est le président de France Télévisions...

A : ...oui, avant qu'il n'intervienne pour confirmer le médiateur...
} 
positive est définie comme la volonté de l'énonciateur $(\mathrm{S})$ de satisfaire la face positive du destinataire $(\mathrm{H})$ ("the want of every member that his wants be desirable to at least some others », Brown \& Levinson [1978: 67]). La politesse négative, elle, correspond à la volonté de $\mathrm{S}$ de satisfaire la face négative de $\mathrm{H}$ (" the want of every 'competent adult member' that his actions be unimpeded by others »).

Ces définitions ont souvent été considérées comme confuses (voir Kerbrat-Orecchioni [2005 : 198]), et elles ont parfois donné lieu à différentes interprétations. Les définitions proposées par Kerbrat-Orecchioni [2005 : 198] pour distinguer "politesse positive » et " politesse négative » sont à cet égard plus opérationnelles. La politesse négative consiste à éviter de produire un FTA, à en adoucir par quelque procédé la réalisation. Quant à la politesse positive, elle consiste à accomplir quelque FFA (Face-Flattering Act ou acte flatteur/valorisant pour les faces), éventuellement renforcé, concept que l'on retrouve chez d'autres chercheurs sous l'étiquette face-enhancing (Sifianou [1995]) ou face-boosting (Bayraktaroglu [1991]).

Dans le cas qui nous concerne ici, la stratégie d'apport d'information nouvelle comme étant de l'information potentiellement connue relève de la politesse négative telle que définie par Kerbrat-Orecchioni, dans la mesure où il s'agit d'éviter - ou tout au moins de minimiser un FTA (constat de l'ignorance de $\mathrm{H}$ ), qui pourra être adouci par le choix de la structure hiérarchisante (aucune présupposition de l'ignorance de $\mathrm{H}$ ) mais également par l'insertion de certains adoucisseurs (voir section 2.4.).

\subsection{La communication de l'acte de politesse}

Le modèle théorique proposé par Brown \& Levinson atteint également ses limites en ce qui concerne la communication (ostensible) de l'acte de politesse lui-même. La question se pose en effet de savoir si $\mathrm{S}$ véhicule, en plus du contenu informationnel lui-même, son intention d'être poli (I am being polite). Cette question est soulevée dès le départ par Brown $\&$ Levinson, qui semblent répondre de façon affirmative dans leur définition de la politesse :

Politeness is then a major source of deviation from [the] rational efficiency [of speaking in accordance with Grice's Maxims], and it is communicated precisely by that deviation $^{8}$. [Brown \& Levinson 1987 : 95]

Selon un tel point de vue, la politesse représente alors une implicature au sens gricéen du terme: "I intend to be polite" "I'm being polite here because I'm sensitive to your face needs" [Fraser 1990]). Cette position est clairement résumée par Pfister [2009 : 3] :

An utterance is polite if and only if:

1. there is an implicature, which is generated because of a violation of a conversational maxims because of concerns of face, and

2. the implicature has the content "I intend to be polite" or "I'm being polite here because I'm sensitive to your face needs".

Si cette conception de la politesse est aisément compréhensible pour l'emploi d'expressions lexicales figées ou de structures grammaticales à visée de politesse telles que s'il vous plaît, merci, pourriez-vous me dire où se trouve la gare?, etc., elle pose problème dans le cas précis que nous soulevons ici. En effet, si l'énonciateur rend l'acte de politesse manifeste lui-même et le véhicule (sous forme d'implicature), alors l'énonciateur commet un FTA. Cela revient en effet à sous-entendre (15), qui si véhiculé, représente nécessairement un FTA :

\footnotetext{
${ }^{8}$ Nous soulignons.
} 
(15) Je te donne cette information que tu ne connais (probablement) pas, mais je fais comme s'il était possible que tu la connaisses en employant une structure hiérarchisante afin de ne pas menacer ta « face ».

Autrement dit, l'énonciateur véhiculerait le message selon lequel il connaît l'ignorance du destinataire, justifiant ainsi l'emploi d'une structure hiérarchisante. Si l'on souhaitait rendre cette implicature explicite, on obtiendrait (13d)-(14c), dont il est assez aisé de percevoir le caractère menaçant (au sens de Brown \& Levinson) pour la face de $\mathrm{H}$ :

\section{(13d) Shirin Ebadi, who I (politely) inform you won the 2003 Nobel Peace Prize, has just arrived in town. \\ (14c) Then I became interested in something called Tai Chi $<,>$ which I (politely) inform you is a Chinese movement form}

Il semble donc qu'il faille, face aux données que nous étudions ici, répondre par la négative à la question soulevée. Nous sommes de ce point de vue en accord avec Sperber \& Wilson, pour qui la politesse n'est pas nécessairement communiquée par l'énoncé produit, de nombreux exemples de politesse n'étant pas manifestes. L'acte de politesse lui-même («je suis poli et je te le dis ») n'est donc pas nécessairement communiqué, car un tel message, véhiculé sous forme d'implicature, reviendrait à commettre un FTA. Ainsi, comme le souligne Pfister [2010], mais aussi Fraser \& Nolen [1981] lorsqu'ils exposent leur contrat conversationnel, un énoncé peut être poli même en l'absence de communication de cette politesse ("an utterance is polite even in the absence of communicated politeness", [Pfister $2010: 6])$.

\subsection{La présence d'adoucisseurs (softeners)}

Le poids (weightiness, W) d'un FTA peut être réduit par la présence d'adoucisseurs (softeners), verbaux ou non-verbaux. Un adoucisseur verbal est une expression ou une construction syntaxique qui vise à réduire l'impact d'un acte qui pourrait s'avérer menaçant pour la face du destinataire. Ainsi l'énonciateur pourra non seulement sourire, incliner la tête (non verbal) mais aussi employer des expressions comme s'il te plaît, excusez-moi afin de limiter l'impact d'une demande ou des structures syntaxiques telles des formes passives comme Ce problème n'a pas été résolu correctement pour signaler à quelqu'un qu'il/elle n'a pas su résoudre un problème [Kerbrat-Orecchioni 2005 : 210-211].

Dans le cas qui nous concerne ici, au-delà de la structure utilisée qui permet de faire passer de l'information nouvelle comme de l'information possiblement connue, l'énonciateur (S) peut avoir recours à d'autres adoucisseurs. En particulier nous aimerions nous attarder sur le cas de l'expression (as) you know - même si you know et as you know ne sont pas équivalents en ce qui concerne leurs emplois en discours, notamment parce que you know est en anglais contemporain plus figé que as you know. Dans les deux cas, et dans le cadre de l'analyse de nos données, ce que semblent dire you know et as you know est: " je te donne l'information mais je sais que tu la connais déjà ». Cet adoucisseur annule alors la présupposition d'ignorance qui constituait un FTA pour le(s) destinataire(s). Ci-dessous, nous considérons tour à tour des exemples faisant intervenir you know puis as you know. 


\subsubsection{L'emploi de you know comme adoucisseur}

Considérons l'exemple (16) :

(16) A: I mean I'm going to see a play next week with an actress friend of mine in it who is now who's doing very well now thank you very much whether

B: oh who's that?

A: Jo Jo Lumley who's you know doing the Revenger's Tragedy

B: oh yes oh yes (ICE-GB s1a-067 184)

B ne semble pas savoir qui est Jo Lumley (ou a feint de l'avoir oublié comme semble l'indiquer les deux oh yes de B qui chevauchent le second tour de A). A lui donne (rappelle ?) le nom de son amie actrice et ajoute de l'information concernant la pièce qu'elle joue actuellement. On peut légitimement se demander pourquoi utiliser l'expression you know, qui semble présupposer que B connaît l'information (statut hearer old selon la terminologie de Prince). Pourquoi en effet véhiculer de l'information si elle est connue ? Il y aurait alors violation de la maxime gricéenne de quantité. L'emploi de you know signifie ici tout autre chose : l'information n'est pas connue de B, mais elle est présentée comme connue, ce qui n'est pas la même chose et relève du phénomène de politesse décrit supra. Afin d'éviter de commettre un FTA, ou tout au moins pour en adoucir l'impact, A présente l'information comme étant déjà connue de $\mathrm{B}$, évitant ainsi de menacer la face de $\mathrm{B}$ dont $\mathrm{A}$ risquerait de présupposer l'ignorance. La réaction de $\mathrm{B}$ (oh yes, oh yes), en chevauchement avec le tour du locuteur $\mathrm{A}$, participe de cette connivence qui vise à préserver la face des deux participants. L'expression figée you know sert alors d'adoucisseur («je te dis même si je sais que tu le sais déjà »).

Le même type d'analyse peut être proposé pour l'exemple (17) :

(17) I mean it is Aspire's job is to raise money <,> for not only the Mike Heafy centre but for research $<,>$ uhm $<,>$ which you know there're many projects that they have on hand $<,>$ uhm $<,>$ (ICE GB s1a-003 070-1)

Dans cet exemple, S, au sein d'une discussion spontanée entre étudiants, explique le rôle de Aspire, organisation qui gère et finance le Mike Heafy centre, et finance également des projets de recherche. Au moyen de la PRA, l'énonciateur informe son destinataire de l'existence de nombreux projets de recherche. Cette information, qui ne semble pas connue du destinataire si l'on se réfère au contexte fourni par le corpus ICE-GB, est donc de l'information nouvelle mais présentée comme partagée, comme le montre l'emploi de you know. Ce faux statut informationnel et la présence de you know permettent de ne pas commettre un FTA, qui consisterait en la présupposition de l'ignorance de $\mathrm{H}$.

L'exemple suivant (18), qui ne met cependant pas en jeu une PRA, est également intéressant à analyser de la même façon :

(18) We've seen a couple of them in practice and they do tend to involve the Procrustean bed $\mid$ you know... everybody who doesn't fit gets a part chopped off

Bien qu'il ne s'agisse pas d'une PRA, la fonction discursive remplie par l'indépendante juxtaposée est exactement celle d'une PRA de pertinence. Nous sommes ici dans le cadre de la communication orale (l'énonciateur est écrivain - en l'occurrence Margaret Atwood - et s'adresse à une journaliste dans le cadre d'une interview enregistrée), registre pour lequel la PRA est quatre fois moins fréquente qu'à l'écrit [Loock 2010 :129-130]. On imagine aisément 
que dans le cadre de la communication écrite, sans la prosodie démarcative qui permet de hiérarchiser les contenus utilisés ici entre les deux propositions, l'indépendante introduite par you know serait reformulée par une PRA :

(18a) We've seen a couple of them in practice and they do tend to involve the Procrustean bed, where, you know, everybody who doesn't fit gets a part chopped off.

L'énonciateur a introduit le SN the Procrustean bed et souhaite préciser la signification de cette expression empruntée à la mythologie grecque. Les destinataires, dans le cadre d'une interview, sont nécessairement multiples (journaliste in praesentia et téléspectateurs in absentia) et nécessairement hétérogènes quant à leurs connaissances. Si pour certains la signification du lit de Procuste représente de l'information hearer old, il est évident qu'elle représentera de l'information hearer new pour au moins une partie des destinataires. La proposition indépendante introduite par you know sert donc bien à niveler l'espace cognitif partagé. L'emploi de l'expression you know, qui signale une digression par rapport au foreground de l'énoncé, permet à l'énonciateur de minimiser le FTA que représente l'apport d'information nouvelle et ainsi d'éviter de passer pour une donneuse de leçon condescendante vis-à-vis de destinataires qui ne sont pas nécessairement aussi initiés qu'elle. L'expression you know fait alors office d'adoucisseur.

Il convient cependant de ne pas être simpliste ici. L'expression you know a en discours différentes fonctions et ne peut se résumer au rôle d'adoucisseur que nous lui attribuons dans les exemples supra dans le cadre d'apport d'information nouvelle pouvant constituer une FTA. Il ne serait pas non plus correct d'affirmer que you know conserve systématiquement son sens littéral, l'expression ayant été grammaticalisée, relevant fréquemment de la relation interpersonnelle. Des travaux récents ont montré que l'expression est en fait fréquemment utilisée afin d'introduire de l'information nouvelle : selon Vincent et al. [2009], seuls 25\% des emplois de you know dans leur corpus sont en lien avec l'idée d'information partagée [Vincent et al. 2009 : 214]. De façon intéressante, parmi les fonctions dégagées pour cette expression en discours, Vincent et al. font état d'un emploi permettant d'introduire de l'information "probablement pas nouvelle pour la majeure partie des destinataires, mais supposée par l'énonciateur comme n'étant pas acceptée à l'instant d'énonciation comme étant vraie » [Vincent et al. 2009 : 216], nous traduisons). L'information est alors imposée, de façon non agressive (Vincent et al. utilisent l'adverbe gently) aux destinataires comme étant de l'information ne prêtant pas à controverse. Ce type d'emplois nous semble être en lien direct avec l'emploi de you know comme adoucisseur de FTA que nous proposons ici, même si dans nos exemples l'information véhiculée est nouvelle dans les faits, en dépit d'une fausse hiérarchisation permettant de la présenter comme étant possiblement connue.

\subsubsection{Le cas de as you know}

L'expression as you know, moins figée que you know, peut également servir d'adoucisseur :

(19) and I think it's dismaying to hear the kinds of things which strike Hilary Steadman who is part as you know of the National Institute which has the unit which has perhaps done the most profound work on comparisons between this country and European countries and indeed other countries uh in this field (ICE GB s2a-031 062) 
Le rôle de as you know, moins grammaticalisé que you know, permet à l'énonciateur (identifié comme lecturer, Director of the National Committee on Education par le corpus ICE-GB, qui identifie par ailleurs la situation d'énonciation comme étant celle d'une conférence) d'introduire de l'information au sujet de Hilary Steadman, discourse new au sein de la situation d'énonciation et dont le statut informationnel hearer new/hearer old ne peut être déterminé avec certitude, l'auditoire étant par définition hétérogène. L'insertion de la PRA de pertinence permet donc de véhiculer de l'information jugée pertinente et possiblement nouvelle pour les destinataires afin de niveler l'espace cognitif partagé. L'insertion de as you know permet de minimiser l'éventuel FTA que constitue l'apport d'information nouvelle.

Nous ne pouvons à cet égard nous empêcher de mentionner un exemple équivalent en français, faisant intervenir comme vous le savez, et prononcé lors de la journée d'étude dont ces actes sont le fruit par l'une des intervenantes, en l'occurrence Madame KerbratOrecchioni, qui après avoir introduit pour la première fois dans le discours la théorie de Brown et Levinson (discourse new) a précisé, au moyen d'un syntagme nominal apposé la nature de cette théorie :

(20) la théorie de Brown et Levinson, la théorie du face work, comme vous le savez.

L'ajout de as you know permet d'annuler toute présomption d'ignorance (information supposée hearer new) devant un auditoire que l'on pourra qualifier d'initié, au sein duquel il n'est toutefois pas complètement impossible d'imaginer certains destinataires pour qui l'information fut nouvelle.

Cependant, et de façon intéressante, as you know, renforcé dans l'exemple (21) infra par I'm sure, peut avoir l'effet inverse, tout comme s'il vous plaît peut être perçu comme un reproche lorsque l'on cherche à attirer l'attention de quelqu'un qui serait peu concentré :

(21) [Contexte : lettre ouverte à visée revendicatrice d'une bibliothécaire à son représentant au Congrès]

Dear Congressperson,

I am writing you today regarding the state of our nation's libraries; specifically the Library of Congress, which, as I'm sure you know, is our nation's oldest federal cultural institution and the largest library in the world. (http://catalogsofbabes.wordpress.com)

C'est ici le caractère initié du destinataire, un représentant du Congrès américain, qui déclenche le FTA, renforcé par as I'm sure you know, qui a donc l'effet inverse d'un adoucisseur. Dans la mesure où il semble acquis que l'information [the Library of Congress/be our nation's oldest federal cultural institution and the largest library in the world] est hearer old pour le destinataire, véhiculer cette information au moyen d'une PRA au sein de laquelle apparait as I'm sure you know déclenche un effet sarcastique assimilable à un FTA.

\subsubsection{Les marqueurs d'hésitation}

Enfin, nous citerons, de façon cependant rapide et sommaire, l'emploi de marqueurs d'hésitation tels que erm.../uh.../well..., qui peuvent fonctionner comme adoucisseurs afin de limiter l'impact d'un FTA. Si ces marqueurs fonctionnent souvent comme remplisseurs (fillers en anglais) dans le cadre de dysfluences ou d'erreurs de performance, permettant à l'énonciateur de gagner du temps, ils peuvent également permettre de rendre une assertion 
moins péremptoire (voir Kerbrat-Orecchioni [2005 : 215]). L'exemple (22) semble illustrer ce même cas de figure :

(22) And the other's about uh this guy called Chomsky $<_{, \text {, }}>$ who's $\boldsymbol{u h}<_{, \text {, }}>$ well one of the world 's most important human beings if you happen to be interested in linguistics $<,>$ (ICE GB s1a-092 103)

Nous ne creuserons pas davantage la question ici ; un travail plus approfondi serait nécessaire afin de dégager de façon précise et rigoureuse le rôle d'adoucisseur de ces marqueurs, par opposition à leurs autres fonctions en discours.

\section{La politesse comme continuum}

La dernière question que nous souhaitons soulever dans le cadre de cet article est la question du continuum qui existerait entre les actes plus menaçants pour la face du destinataire et les actes moins menaçants. Partant du constat que l'on peut être plus ou moins poli, des chercheurs comme Fraser \& Nolen [1981 : 97], par exemple, ont proposé que la politesse soit un continuum.

Ci-dessous nous explorons deux hypothèses selon lesquelles le FTA est plus ou moins marqué : (i) selon le statut informationnel réel de l'information véhiculée, mais aussi (ii) selon le type de structure hiérarchisante utilisée. Ces hypothèses vont donc dans le sens de l'existence d'un continuum.

\subsection{L’importance du FTA selon l'information véhiculée : le « fame effect »}

Comme le montrent les exemples mentionnés jusqu'ici, tout contenu informationnel ne peut pas être évalué par l'énonciateur comme étant hearer old ou hearer new pour le(s) destinataire(s) avec certitude, d'où la création de la catégorie des indéterminables mentionnée supra. Il n'en reste pas moins que l'énonciateur peut évaluer le caractère plutôt hearer new ou plutôt hearer old en fonction de la saillance ou de la notoriété de cette information.

Considérons en effet la série de paires d'exemples suivants, où chaque paire consiste en un nom propre suivi d'un syntagme nominal apposé simple pour le premier exemple et d'une relative appositive (PRA) pour le second, afin de spécifier l'identité du référent dénoté par le nom propre :

(23) Barack Obama, the president of the United States, has arrived in Europe. (23a) \#Barack Obama, who is the president of the United States, has arrived in Europe.

(24) Bill Clinton, the former president of the United States, will attend an international seminar on AIDS and SARS.

(24a) \#/??Bill Clinton, who is the former president of the United States, will attend an international seminar on AIDS and SARS.

(25) Angela Merkel, the German chancellor, on Friday described Barack Obama's presidency as a "unique opportunity".

(25a) \#/??Angela Merkel, who is the German chancellor, on Friday described Barack Obama's presidency as a "unique opportunity".

(26) Djokovic, the Australian Open champ, earned the top spot based on his victory over Davydenko during the week. 
(26a) ?Djokovic, who is the Australian Open champ, earned the top spot based on his victory over Davydenko during the week.

(27) Martin Townsend, editor of the Sunday Express, has made a personal appeal for her safe return.

(27a) Martin Townsend, who is the editor of the Sunday Express, has made a personal appeal for her safe return.

(28) Edgar Griffin, the father of the BNP leader Nick Griffin, was sacked as a vice-president of the Duncan Smith campaign in Wales.

(28a) Edgar Griffin, who is the father of the BNP leader Nick Griffin, was sacked as a vice-president of the Duncan Smith campaign in Wales.

De façon intéressante, on note qu'en dépit de structures identiques (nom propre de personne suivi d'un SN apposé simple (23-28) puis d'une PRA (23a-28a)), la manipulation qui consiste à remplacer le SN apposé par une PRA n'est pas pragmatiquement recevable de façon systématique et homogène. Dans des travaux précédents, nous avons montré qu'il s'agissait là de ce que nous avons appelé en anglais le «fame effect» [Loock 2009] : plus l'information a de chances d'être connue par le destinataire, moins la reformulation par une PRA, qui rend le lien d'identification explicite, est acceptable, par opposition au SN apposé (voir section 3.2) :

+ HEARER OLD/- PRA

(23b) Barack Obama is the (newly elected) president of the United States.

(24b) Bill Clinton is the former president of the United States.

(25b) Angela Merkel is the German Chancellor.

(26b) Djokovic is the Australian Open champ.

(27b) Martin Townsend is the editor of the Sunday Express.

(28b) Edgar Griffin is the father of the BNP leader Nick Griffin.

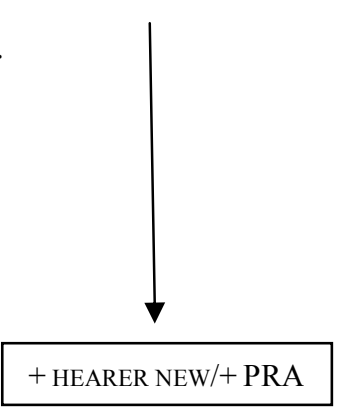

Dans ces conditions, l'emploi de la PRA représente également un FTA plus important au fur et à mesure que l'information est connue (+ hearer old). La présomption d'ignorance est plus impolie s'agissant de Barack Obama que d'Edgar Griffin, par exemple. Ceci signifie qu'il existerait différents degrés d'impolitesse et argue en faveur de la politesse/l'impolitesse comme continuum.

\subsection{L'influence du choix de la structure}

Egalement, il semblerait que l'importance du FTA soit dépendante de la structure hiérarchisante utilisée. Nous avons listé plus haut (exemples (8) à (12)) les structures autres que la PRA permettant de hiérarchiser les contenus informationnels. Les contraintes régissant le choix entre ces différentes structures sont nombreuses, mais certaines peuvent être mises en relation avec le degré de présomption d'ignorance du destinataire. Ainsi, le syntagme nominal apposé (Barack Obama, the president of the United States) ne présuppose rien quant au caractère hearer new/hearer old de la relation [Barack Obama/be the president of the US], ce qui explique sa fréquence dans le registre journalistique par opposition à l'inexistence de l'exemple avec la PRA et ce qui explique la recevabilité des exemples (23) à (28) supra. Le SN apposé pourra alors être utilisé quel que soit le statut informationnel de la relation d'identification entre les deux SN en apposition, alors que ce n'est pas le cas de la PRA. 
De la même manière, l'emploi d'une parenthétique phrastique représente un FTA plus important que l'emploi d'une PRA dans la mesure où il s'agit d'une interruption trop manifeste du discours :

(10e) Shirin Ebadi - she's the 2003 Nobel Peace Prize winner - has just arrived in town.

(11d) Then I became interested in something called Tai Chi - it's a Chinese movement form

Le fait que l'énonciateur interrompe son discours - il y a rupture de la syntaxe - afin d'introduire de l'information à visée de pertinence au sujet de Shirin Ebadi ou du Tai Chi représente une digression trop explicite, contrairement à l'emploi d'un SN apposé simple ou d'une PRA, et représente ainsi un FTA. Ce type de FTA est alors plus important que l'emploi d'une PRA, qui, intégrée à la structure syntaxique de la phrase, représente une digression moins marquée.

Ceci nous permet de conclure que la structure utilisée correspond à des FTA plus ou moins marqués : neutralisés avec un SN apposé, marqués avec une PRA, encore plus marqués avec une parenthétique phrastique. Ceci argue également en faveur de l'existence d'un continuum pour la politesse/l'impolitesse : l'emploi d'une PRA est moins impoli que l'emploi d'une parenthétique phrastique afin d'introduire de l'information périphérique nouvelle à visée de pertinence.

\section{Conclusion}

Dans cet article, nous avons souhaité montrer que la simulation d'une hiérarchisation informationnelle est gouvernée par des considérations liées à la politesse. Faire passer de l'information nouvelle pour le destinataire comme de l'information potentiellement connue évite de présupposer l'ignorance de ce dernier, et donc de commettre un FTA selon la terminologie de Brown \& Levinson. Pour ce faire, l'énonciateur a recours à une structure hiérarchisante comme la relative appositive ou encore un SN apposé ou une parenthétique phrastique, le choix entre ces différentes structures courantes étant - toutes choses étant égales par ailleurs - gouverné par le statut informationnel réel du contenu véhiculé. L'énonciateur peut également insérer des adoucisseurs comme you know ou as you know qui permettent de présenter de façon paradoxale de l'information nouvelle tout en annonçant explicitement son caractère connu.

$\mathrm{Au}$ travers de cet exemple spécifique, nous avons pu explorer différents concepts théoriques concernant la politesse linguistique. Ainsi, si le cadre proposé par Brown \& Levinson s'avère particulièrement opérant pour décrire le type de données étudiées ici, il montré également ses limites dans ce cas précis s'agissant de la communication de l'acte de politesse lui-même.

\section{Bibliographie}

BAYRAKTAROGlu Arin, "Politeness and interactional imbalance", International Journal of the Sociology of Language ${ }^{\circ} 93,1991: 5-34$.

Brown Penelope \& LEVINSON Stephen, "Universals in language usage: Politeness phenomena", in Goody E. (ed), Questions and politeness, Cambridge, Cambridge University Press, 1978 : 56-289. 
Brown Penelope \& Levinson Stephen, Politeness: Some universals in language usage, Cambridge, Cambridge University Press, 1987.

FrASER Bruce, "Perspectives on politeness", Journal of Pragmatics $n^{\circ} 14,1990: 219-236$.

FrASER Bruce \& NOLEN William, "The association of deference with linguistic form", International Journal of the Sociology of Language $\mathrm{n}^{\circ 2}$ 27, 1981 : 93-109.

GOFFMAN Erving, Interaction ritual: essays in face-to-face behavior, Random House, Aldine Transaction, 1967.

JARY Mark, "Relevance Theory and the Communication of Politeness", Journal of Pragmatics n०30, $1998: 1-19$.

Kerbrat-Orecchioni Catherine, Le discours en interaction, Paris, Armand Colin, 2005.

LEECH Geoffrey, Principles of Pragmatics, London, Longman, 1983.

LOOCK Rudy, "Les Fonctions discursives des propositions subordonnées relatives appositives en discours », Anglophonia n 12, 2003 : 113-131.

Loock Rudy, "Appositive Relative Clauses and their Functions in Discourse", Journal of Pragmatics $\mathrm{n}^{\circ} 39,2007: 336-362$.

LoOcK Rudy, "How the choices of speakers between allostructures can be explained by their assumptions about their addressees' states of knowledge: the case of relevance-oriented, non-restrictive noun modifiers", Atelier linguistique du CRISCO "Ce que le texte fait à la phrase" (Université de Caen), 3-4 décembre 2009: actes à paraître.

LoocK Rudy, Appositive Relative Clauses in English: Discourse Functions and Competing Structures, Amsterdam/Philadelphia, John Benjamins, 2010.

PFister Jonas, "Is there a need for a maxim of politeness?", Journal of Pragmatics n42, 2010, sous presse.

Pince Ellen F., "Toward a Taxonomy of Given/New Information”, in Cole P. (ed.), Radical Pragmatics, New York, Academic Press, 1981 : 223-254.

Prince Ellen. F., "The ZPG Letter: Subjects, Definiteness, and Information-Status", in Thompson S. \& MANN W. (eds), Discourse Description: Diverse Analyses of a Fund Raising Text, Philadelphia, John Benjamins, 1992 : 295-325.

SifiANOU Maria, "Do we need to be silent to be extremely polite?: Silence and FTAs", Applied Linguistics n5, 1995 : 95-110.

SPERBER Dan \& Wilson Deirdre, Relevance: Communication and Cognition, Oxford, Blackwell, (1986) 1995.

Vincent Sophie, Darbaky Sarah \& Mettouchi Amina, "The grammaticalization of you know. From shared knowledge to control over the co-speaker", English Text Construction $n^{\circ} 2: 2,2009: 209-227$.

Watts Richard J., Politeness, Cambridge, Cambridge University Press, 2003. 